\title{
Performance Enhancement of LTE through Interference Reduction Techniques
}

\author{
M.Sugacini, N.Kumaratharan* \\ Department of IT,Sri Venkateshwara College of Engineering, India \\ *Corresponding Author: kumaratharan@rediffmail.com
}

Copyright () 2014 Horizon Research Publishing. All rights reserved.

\begin{abstract}
Long Term Evolution (LTE) is the standard for wireless communication of high speed data and mobile terminal. Among the current emerging technologies like LTE based on orthogonal frequency division multiple access (OFDMA) technique, it found to be more attractive for high speed wireless communication. However co-channel interference (CCI) from the neighbouring cells is one of the limiting factors that degrade the LTE system. Fractional frequency reuse (FFR) technique is proposed to overcome the same as it is suitable for idealized cellular layout since power is not considered in this scheme. In this paper FFR is generalized and sub band allocation among user is made in optimal manner which increases the performance of the system.
\end{abstract}

Keywords Co-channel interference, LTE, FFR

\section{Introduction}

LTE is Third Generation Partnership (3GPP) enhancement to the cellular system in use. The purpose of developing this is to keep 3GPP system competent enough for decades to come. LTE is designed to have wider channels up to 20MHZ, with low latency and packet optimized access technology. The peak data rate envisaged got LTE is 100 Mbps in downlink and 50Mbps in the uplink, it promising features like bandwidth scalability and both FDD and TDD duplexing methods. Orthogonal frequency division multiple access is a multi user version of OFDM digital modulation scheme. Multiple accesses are achieved in OFDMA by assigning subsets of subcarriers to individual users at a time. These subcarriers are assigned based on the demands of the users. This allows low data rate transmission from several users. Most of the current emerging cellular system based on OFDMA. The spectrum divided into sub-carriers and cluster of sub-carrier allocated to different subscriber. However in certain distance the need to reuse the spectrum. Each spectrum orthogonally allocated different users. LTE achieve high data rate and throughput because of OFDMA system. The performance enhancement limited by the co-channel interference. The spectrum reuse will be allowed at the certain distance $\mathrm{D}=\mathrm{R} \sqrt{2} \mathrm{~N}$, where $\mathrm{R}$ is the radius of the cell, $\mathrm{N}$ is the number of cell in the particular coverage. By this distance effect of CCI will be less; if the two users having same frequency are nearer the effect of CCI will be high. Compared to center user, the edge user will be affected by CCI and their by causing poor quality of service (QoS), low throughput, and high delay. In the current mobile communication, the frequency resource is divided into non-overlapping spectrum bands and is assigned to each region of the cell. Co-channel interference is exists between any neighboring cell in LTE cellular networks due to sharing of same channel which degrades the system performance. To improve the efficiency of spectrum the frequency reuse methods are adopted. However on other hand this method leads to co-channel interference as the same set of frequency is used by several cells in the network.

Some interference cancellation scheme has been proposed to reduce the inter cell interference (ICI) in OFDM system [1]. The allocation of sub-band is achieved through semi-static [2]-[3] network co-ordination, the division of sub-band taking into account of traffic load and data requirement. The coordination of transmission modes of multiple base stations is called beam forming techniques and this approach to reduce the amount of interference in networks [5]. To achieve high throughput for the cell edge user FFR reuse technique is employed in the literature of [6]. They minimize the effect of CCI by dividing the total available bandwidth into two sub-bands. The reuse-1 used for cell center user and reuse- 3 used for the cell edge user and it is different from other neighboring cells. In LTE, the resource of physical resource block (PRB) consists of 15 subcarriers that is equal to $15 \mathrm{KHZ}$ space division between the subcarriers is constant. The PRB used to transmit long range of data. Once the channel reserved for the user, the resource block (RB) are allocated for data's, which consist of 12 subcarriers. In FFR scheme there is various approach to allocate the RB among users. This scheme has some limitations for the user if they are not allocating the sufficient bandwidth. Typically each RB is reserved for the frequency allocation. FFR technique can be applied for both uplink and downlink transmissions. In FFR scheme, the division of 
sub-band is fixed. This technique is suitable for static cellular layout. Mostly FFR consider fixed partitioning of resource for cell center and edge users. Fixed thresholds are used in terms of distance and SINR.

In hybrid CCI mitigation scheme using both partial frequencies, handover technique and they assume a fixed SINR metric to separate the user into center and edge, while the bandwidth partitioning of a cell has not been described for different SINR thresholds. The impact of scheduling strategies and increase in number of users in a cell based on the design of the optimal SINR threshold but it has not shown the improvement on cell edge performance. The fixed SINR threshold value used for bandwidth partitioning on the cell edge and center performance for both real time and best effort traffic. Normally bandwidth required for the users depend on the location. If the amount of resource required by users known, one can find the number of users supported in a cell. The allocation pattern ensures that sub-band of a cell-edge zone is not reused in any of the neighboring cells. The cell varies greatly in the occurrence of interference, causing the difficulties in the standard FFR. Finally scalability becomes issues and allocation of sub-band is fixed, we can't give assurance for throughput of users, because users can dynamically change.

Flexible spectrum reuse schemes have been proposed such as soft frequency reuse (SFR) [4] in the 3GPP systems. The SFR can overcome severe CCI problems from adjoining cells at cell edge region, by emphasizing a part of the available spectrum for edge users. The conventional [7]SFR scheme is based on static reservation, which partitions the resource region into two orthogonal portions, one uniquely dedicated to users in the cell and the other uniquely dedicated to other users. This kind of resource allocation which restricts to each user can ensure in SINR gain in average manner. From users perspective integer frequency reuse cab be regarded as a special case of FFR. In integer frequency reuse (IFR) all RB allocated to a cell can be used anywhere in the cell without any specification of users locations. Each cell listens on every secondary sub channel all the time and support flexibility with non uniform user or traffic distribution.

\section{Generalized and Optimized FFR Model}

Generalized and optimal FFR scheme is proposed, to overcome the limitations of standard FFR and dealing with real time networks instead of irregular layout. By first step total available bandwidth is divided in any number of sub-bands and that is unique from the neighboring cell. Doing so it is useful for avoiding interference in cells with many surrounding and the number of sub-band used for edge users must be specific because of division of more sub-band sensitive to interference so the partitioning the sub-band based on the throughput of cell. It is combination of static and dynamic FFR scheme, each base station periodically calculate the throughput if it less than SINR threshold then the division of sub-band will be changed dynamically to satisfy the edge users.

The extending standard FFR, assigning power is part the of optimization framework. This scheme allows the power vary by cell in order to achieve higher data rate. By considering the power assignment and allocation of sub-band the performance of system is calculated.

In the system model the service area is denoted as pixel and granularity of creating the sub-band is high and edge zone may use multiple sub-bands. In addition, the power varies by cell. For example cell one has more edge sub-bands allocated than other two cells, the edge zone is less sensitive interference. And the proposed distributed and coordinated resource allocation technique to self organizes the reuse pattern. In this scheme each cell independently and dynamically allocates the resource block and transmits power to the users so power assigned to each user is minimized, while user throughput is maximized. More over each cell inform neighboring cells of the resource block that have been scheduled for message passing. The optimal allocation done by allocating the frequency, proposed mechanism assumes a topology and to find the optimal deployment, the mechanism uses an algorithm, which divides each cell into two regions, assuming 25 resource block are applied .In the first cell entire resource block allocated to inner region and second cell 24 resource block allocated to inner region and remaining allocated to outer region, third cell 23 resource block allocated to inner region and remaining to outer region.(Figure1)

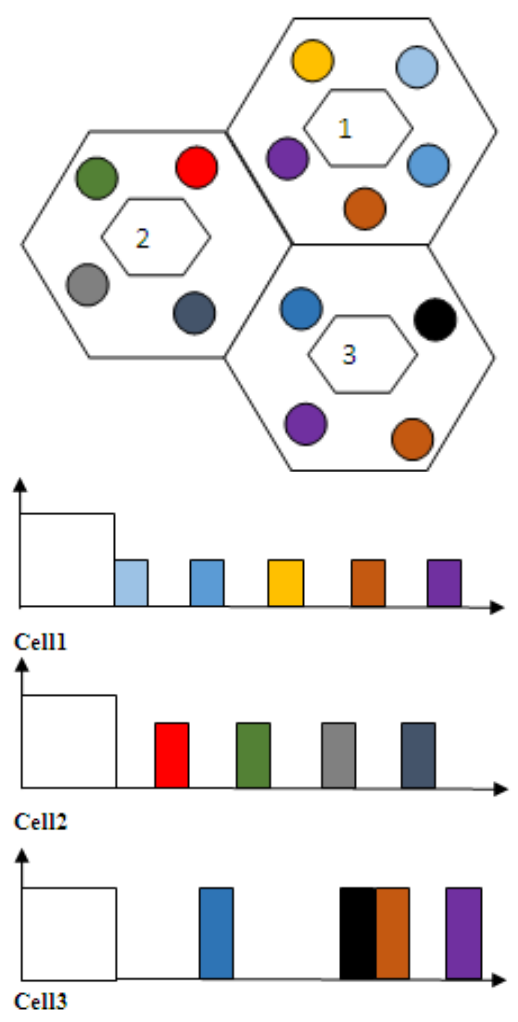

Figure 1. Proposed Frequency band allocation 
The basic elements for the system model and notations are presented here and consider the set of cells in the particular coverage and it is denoted as $\mathrm{C}=\left(\mathrm{c}_{1}, \mathrm{c}_{2} \ldots \ldots \ldots \mathrm{c}_{\mathrm{n}}\right)$. The coverage area represented by regular hexagon of a large number of pixels $J=\left(\mathrm{j}_{1} \mathrm{j}_{2} \ldots \ldots\right)$. The total gain between the cell and the pixels denoted by the $\mathrm{G}_{\mathrm{ij} . .}$. The cell is divided into center zone and edge zone, in which center zone is prone to interference and edge zone is sensitivity to interference from the surrounding cells. The total transmit power of cell antenna is denoted by $\mathrm{p}^{\text {Tol }}$. The entire bandwidth is divided for center user and edge user, center user is denoted as $\mathrm{B}_{c}$, edge user is denoted as $\mathrm{Be}$. Let $\mathrm{K}$ denote the number of edge sub-band $\mathrm{K}=\left(\mathrm{k}_{1}, \mathrm{k}_{2} \ldots \ldots\right) \mathrm{B}_{\text {sub }}=\mathrm{B}_{\mathrm{e}} / \mathrm{K}$. In the generalized $\mathrm{FFR}$ number sub-band allocated to each cell that is orthogonal to neighboring cells. Consider for sub-allocation and power assignment in each cell, now we have three base stations. In first base station the number of sub-band is 4 , second base station the number of sub-band is 5and finally third base station the number of sub-band in 4 . Now we are going to calculate the SINR vale for each cell

$$
\mathrm{SINR}=\mathrm{p}_{\mathrm{i}} \mathrm{g}_{\mathrm{ij}} / \sum \mathrm{p}_{\mathrm{h}} \mathrm{g}_{\mathrm{hj}}+\sigma_{\mathrm{j}}
$$

Where, $g_{i j}$ gain between cell antenna and pixel. Let us consider uniform power assumption over the edge sub-bands in each cell. The power used for cell edge zone is denoted by $p_{i}$ and optimization value selected from the set of power levels $\left(\mathrm{p}_{1, \mathrm{p}_{2} \ldots \ldots . . .}\right)$. The power assign represented by the following vector $p_{L}=\left(p_{1}, p_{2}, \ldots \ldots \ldots \ldots . . . p_{c}\right)$. The maximum value of power depends on the number of sub-bands used in cell, and then sub-band can range between one and $\mathrm{k}$ in this scheme. Assigning power value to cell is the product between the value of $p_{i}$ and number of sub-band that does not exceed the value of $\mathrm{p}_{\mathrm{L}}$. By using bandwidth partitioning ratio $\alpha$ we going to divide the spectrum for the cell center and cell edge zone

$$
\alpha=\mathrm{P}_{\mathrm{Abc}}
$$

$\mathrm{P}_{\mathrm{Abc}}$, is the averaged probability of selecting band. The approach to partitioning the bandwidth in the cell is by finding the probability of a user to be in center band. Therefore the ratio of bandwidth allocated to center users is equal to the probability of a user to be at that band. This method uses the ratio between the average bandwidth required for a cell center user and average bandwidth required by each user throughout the cell. Bandwidth partitioning ratio $\alpha$ is obtained here as

$$
\alpha=b_{u A c}
$$

Where $b_{u A c}$ is the area bandwidth required for a center band, BW the average bandwidth required by each user throughout the cell. The interference that occurs comes from separate sets of downlink in the inner and outer region. A transmission in an inner region that is fixing specific frequency band causes interference only to inner users of others cells that are fixed the same band and it is important to distinguish two categories of BSs. All interfering BSs transmitting to inner region users on the same sub-band as user $\mathrm{x}$ and second consists of all interfering BSs to cell edge users.

The capacity of user $\mathrm{x}$ on subcarrier $\mathrm{n}$ can be calculated by the following

$$
\mathrm{C}_{\mathrm{x}, \mathrm{n}}=\Delta \mathrm{f} \cdot \log _{2}\left(1+\mathrm{SINR}_{\mathrm{x}, \mathrm{n}}\right) \cdot \mathrm{P}_{\mathrm{i}}
$$

Where, $\Delta \mathrm{f}$ refers to the available bandwidth for each subcarrier divided by the number of users that share specific subcarrier, Pi refers to power allocated to user $\mathrm{x}$. Moreover, the throughput of the user $\mathrm{x}$ can be expressed as

$$
\mathrm{T}_{\mathrm{x}}=\sum \beta_{\mathrm{x}, \mathrm{n}} \cdot \mathrm{C}_{\mathrm{x}, \mathrm{n}}
$$

Where, $\beta_{\mathrm{x}, \mathrm{n}}$ represents the sub carrier assigned to user $\mathrm{x}$.

\subsection{Search Strategy}

The aim of applying generalized FFR to large network, consider local search algorithm and finding high quality solution time efficiently to system performance. A local search algorithm finds solution improvement by repeating the modification to the current solution and evaluating the outcome. The solution strategy is defined by the operation used in making trial modifications. Solution generated by the modification operation is considered neighbors to the current one, and the definition of the modification operation is also known as the neighborhood structure.

The algorithm stops when no improvement can be obtained by solution modification that is the current division of sub-band is locally optimal in respect of its neighborhood. Apply a type of greedy algorithm that assigning single sub-band to each cell to obtain solution. The algorithm goes through all cells one by one, for each cell the total throughput among the cell edge pixel is completed for each of the sub-band is $(1 \ldots \ldots . . K)$ with same power as reuse-1. Once assigning a sub-band, the allocation for the cell in the initial solution is fixed when considering the remaining cells. The algorithm tends to select the sub-band in which having least interference problem. The search is repeated until the current allocation is locally optimal in every cell. Let the current solution be $\mathrm{C}=\left(\mathrm{C}_{1 \ldots \ldots} \ldots \mathrm{C}_{\mathrm{k}}\right)$ and $\mathrm{P}=\left(\mathrm{P}_{1 \ldots \ldots} \ldots \mathrm{P}_{\mathrm{i}} \ldots \ldots . . \mathrm{P}_{\mathrm{c}}\right)$. The task is to find the new optimal sub-band allocation and power assignment for each cell. Suppose that power assignment is independent from sub-band allocation. Then the allocation of sub-band dynamically changed does not have any interference.

If the sub-band added to the entire cell then the throughput will grow decrease in re use of sub band. Consider now the connection between sub-band and power assignment and the algorithm can be analyzed as follows.

Step 1: Find the best number of sub-band using current solution

Step 2: Calculate the SINR change for the current cell

Step 3: Repeat the steps1-2 for all cells

Step 4: Find the highest throughput improvement among all the cells

Step 5: If the throughput is improved among the cell edge then no change in partition of sub-band 
The optimized average cell edge throughput for various values of $\mathrm{K}$ is assumed and compared the results to reuse-1. From the fig2 shows that if number of band is higher than increase in data rate. It is clear from that commonly used reuse-3 is not the best for an irregular cell layout. The optimized FFR performance tends to increase with $\mathrm{K}$. This behavior is expected, because division of sub-band is high then gives granularity in resource allocation. There is no strict monotonicity. The reason is that the solution returned by the algorithm. By definition, all sub-bands are allocated to cell edge as well as cell center in reuse-1. Therefore performance is not dependent on value $\mathrm{K}$

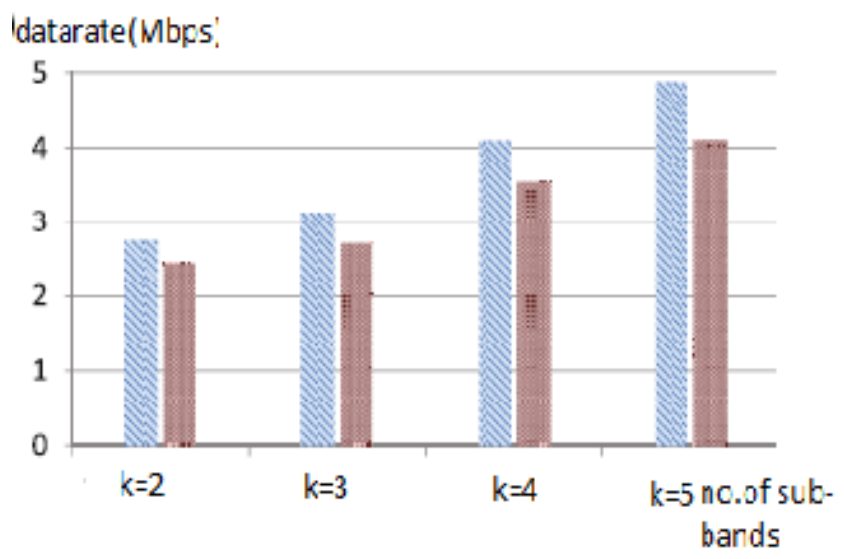

Figure 2. Performance Comparison of value $\mathrm{K}$

Thus considering larger area as cell edge brings down the average throughput. This is because a high threshold means that larger areas are considered as cell edge, although parts of these areas as bandwidth sensitive rather than interference sensitive. The throughput loss of this bandwidth sensitive area significantly decreases the cell edge throughput. That standard FFR will not perform better than optimal FFR with $\mathrm{K}=3$, the standard FFR cannot directly applied with fixed frequency reuse pattern, reuse- 3 the number of neighboring cells varies greatly over the area. In general, increasing in $\mathrm{k}$ implies that more candidate allocation solutions must be evaluated in the search algorithm, and enlarging the edge area size leads to higher number of sub-band, setting $\mathrm{k}$ between 3 and 6 achieves a good balance between the performance in throughput and computational effort. In general the choice of $\mathrm{k}$ depends on the network characteristics and finding a proper value is not trivial. In network planning algorithm can be applied to a sub set of cell edge, to provide fast performance evaluation and thereby suggestions on proper sub-band division scheme. It is showed that optimally selecting the band and dividing the sub-band based on throughput of cell edge users enhance the performance of LTE system. Low SINR threshold leads to be small cell edge areas with very high interference sensitivity and therefore more improvement in optimal FFR. Another factor having a strong influence on performance is the relation between $B_{e}$ and $B_{c}$, setting bandwidth values is network specific and to a large extent, the optimal choice depends on the user distribution as well as the performance target. For optimal FFR enlarging or shrinking the edge bandwidth $B_{e}$ does not change the optimal sub-band allocation. Hence can easily observe the trade-off between the cell edge versus the cell center throughput loss.

\section{Simulation Results}

The following (Table 1) results show the performance of the OFDMA system using generalized FFR. The fixed SINR value used as threshold for dividing the sub band. By doing this data rate, throughput of the system has been enhanced. The results are based on the implementation of random reuse-1, reuse- 3 among the cell .The input parameters chosen for the implementation of reuse-1 for cell center user and reuse-3 for cell edge user in order to simulate results of throughput and data rate by various SINR values.

Table 1. Simulation parameters

\begin{tabular}{|c|c|}
\hline Cellular layout & Hexagon \\
\hline System bandwidth & $15 \mathrm{MHZ}$ \\
\hline No.of subcarriers & 300 \\
\hline No.of eNb & 8 \\
\hline Sub carriers spacing & 15 \\
\hline SINR threshold & $0-100$ \\
\hline Antenna type & Omni \\
\hline Propagation mode & OFDMA \\
\hline
\end{tabular}

The simulations results shows performance of the proposed system and it is executed analyzed using NS2 version 2.31. The available bandwidth is shared among neighboring cells based on the reuse factor. Cell center users use the frequency band $\mathrm{fl}$ while the cell edge user is allocated with $1 / 3^{\text {rd }}$ of the rest available bandwidth. The goal of this work is to investigate the impact on dividing available spectrum into number of sub band and optimally allocating the sub band to the edge users.

\section{THROUGHPUT VS DELAY}

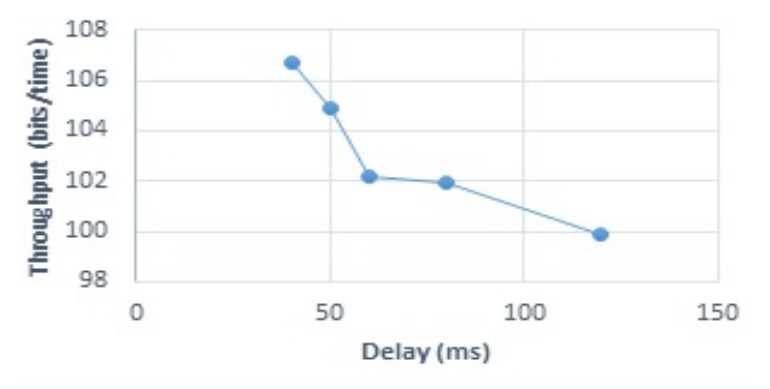

Figure3. Throughput compared vs Delay

Fig 3 shows the relationship between throughput vs delay. If interference occurs in the cell delay of transmitting packet for users so by avoiding the interference throughput will be more. From the figure it is evident that the delay is increased with when the throughput is increased. 
Fig 4 provides the correlation between data rate and SINR. The figure clearly shows that the data rate is increased with higher SINR values due to less interference.

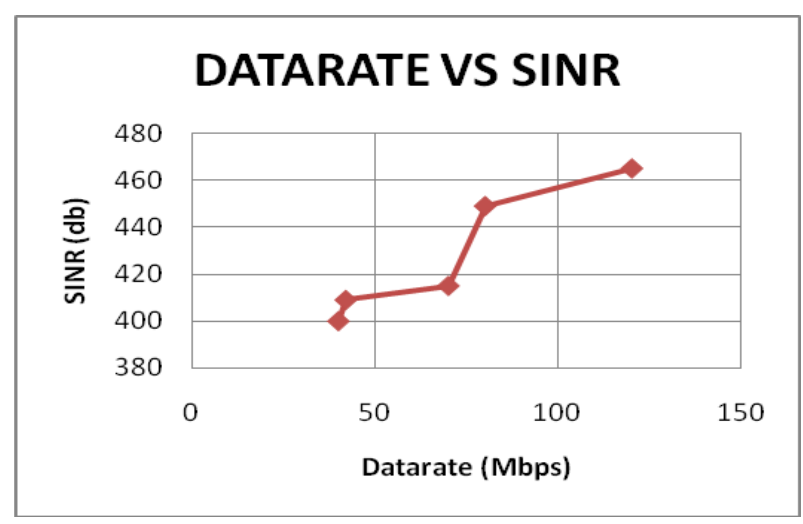

Figure 4. Data rate performance over SINR

Fig 5 provides the link between Throughput and SINR. The figure shows that if SINR value is higher than parallel throughput value also get increased

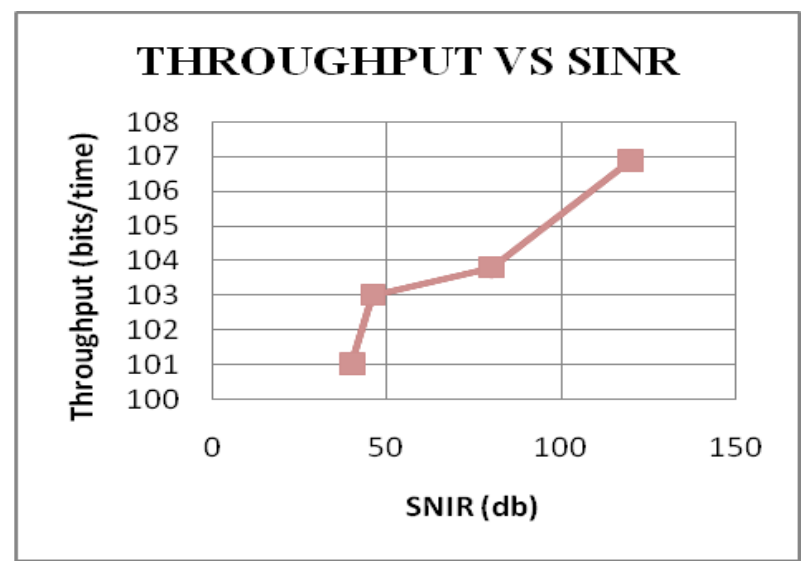

Figure 5. Throughput compared with SINR

\section{Conclusion}

To overcome the limitations of standard FFR and to address the performance of FFR in large scale networks with irregular cell structure, generalized FFR scheme was presented and also finding the optimal sub-band allocation among users was described. By doing this enhancement of system performance of LTE especially for cell edge users throughput was achieved. For highly interference sensitive cell edge zones, interference is minimized by sub-band isolation, where as for the other cell edge zones more bandwidth is allocated and this leads to better performance.

\section{REFERENCES}

[1] Defeng David Huang and Khald(2005), "An interference cancellations scheme for frequency offsets corrections on OFDMA sytem ," in proc .IEEE Transcation Communication,pp.1-5

[2] Yikang Xiang,Jijun Luo(2006), "Inter cell interference mitigation through flexible resource reuse in OFDMA based communication networks," in proc. of the $15^{\text {th }}$ European wireless conference, Paris, France, pp.162-166

[3] 3GPP R1-060368(2006),"Performance of inter cell interference mitigation with semi static frequency planning for EUTRA downlink, “Texas Instrument, pp.1-5

[4] Zheng Xie,Bernhard Walke(2007)," Enhanced fractional frequency reuse to increase capacity of OFDMA systems," in computer network, Germany,volume 314-325

[5] Matthias Kaschub, Christian M.Blankenhorn, Christian M.Muller and thomas(2007)," Interference mitigation by distributed beam forming optimizations," Universitat Stuttgart, Institute of communication Networks and computer engineering,pp.1-7

[6] A.L.Stolyar and H.Viswanatham(2008)," Self-organizing Dynamic fractional frequency reuse in OFDMA systems," in proc .INNFOCOM 2008. The $27^{\text {th }}$ conf.Computer Communiations.IEEE,Bostan Volume.691-699

[7] Bujar Krasniqi, Martin Wrulich and Christoph(2009)," Network load dependent partial frequency reuse for LTE," in proc. IEEE, Institute for Communications and radio frequency engineering Christian doppler laboratory,pp.1-5

[8] Zhang Huil,Xu Xiao-dong,L1 jing(2010)," Performance pf power control in inter cell interference for frequency reuse," The journal of china universities of post and telecommunications, China,pp.37-4. 